\title{
Health Professionals für das 21. Jahrhundert: neue Wege in der Ausbildung
}

\author{
Ein von The Lancet in Auftrag gegebener Bericht zeigt, dass eine nach Beruf und \\ Spezialisierung aufgesplittete Ausbildung am Bedarf vorbeigeht. Gefordert werden \\ systematisches gemeinsames Lernen aller Berufsgruppen auf Basis lernfeldorien- \\ tierter Curricula und eine Neuausrichtung der Akkreditierung.
}

\section{Beat Sottas}

Stiftungsrat, Mitglied des Leitenden Ausschusses Careum Stiftung, Zürich
1 Originaltext unter www. stiftung-careum.ch/news bzw. www.globalcommehp. com/index.php?option $=$ com_content $\&$ view $=$ article \&id=8\&Itemid $=13$ Die deutsche, von der Careum-Stiftung übersetzte Fassung wird ab April in gedruckter Form vorliegen und auf der Website zum Download bereitstehen. Die Strategie wird danach an verschiedenen öffentlichen Anlässen mit Bildungsregulatoren und Bildungsstätten diskutiert.

Korrespondenz: Dr. Beat Sottas Careum Stiftung Pestalozzistrasse 3 CH-8032 Zürich Tel. 0432225029

beat.sottas@careum.ch
Vor 100 Jahren hat Abraham Flexner in einem wegweisenden Report dazu aufgefordert, die medizinische Ausbildung auf wissenschaftliche Grundlagen zu stützen und in die Universitäten zu integrieren. Dieser Strategiewechsel hat einen Wissenszuwachs ermöglicht, der zur Verdopplung der Lebenserwartung beigetragen hat.

$\mathrm{Zu}$ Beginn des 21. Jahrhunderts wird allerdings deutlich, dass längst nicht alles gut ist: Im Gesundheitsbereich wachsen die Ungleichheiten. In und zwischen den Ländern schwindet die Chancengleichheit; die sich öffnende Kluft belegt das kollektive Versagen bei der gerechten Nutzung der Fortschritte. Gleichzeitig werden die Errungenschaften durch neue Herausforderungen bedroht: neue Infektionskrankheiten, neue Umweltrisiken und Verhaltensweisen sowie demographische und epidemiologische Umbrüche.

Das Personal ist die wichtigste Ressource eines jeden Gesundheitssystems. Die Leistungsfähigkeit und die Qualität der Versorgung stehen in einem direkten Verhältnis zur Anzahl der verfügbaren Health Professionals und ihrem Qualifikationsniveau. Alle Gesundheitssysteme kämpfen mit ähnlichen Herausforderungen, steigender Komplexität sowie hohen Kosten und richten zusätzliche Forderungen an die Gesundheitsfachleute. Obwohl weltweit jedes Jahr rund eine Million Health Professionals ausgebildet werden, deckt dies längst nicht den steigenden Bedarf.

100 Jahre nach dem Flexner-Report wurde Ende November 2010 in Boston der unter dem Patronat der renommierten Zeitschrift The Lancet entstandene Bericht «Health professions for a new century: transforming education to strengthen health systems in an interdependent world» [1] vorgestellt. Anlass zur Sorge gibt nicht nur die Anzahl und Verteilung der Health Professionals. Die Lancet-Kommission leitet aus ihrer Analyse Massnahmen für wesentliche Kurskorrekturen und Neuorientierungen in der Ausbildung von Medizinern und anderen Health Professionals ab, damit sie ihre Aufgaben im 21. Jahrhundert bedarfsgerecht ausüben können.

Die Autoren zeigen auf, dass die heute vorherrschenden, nach Beruf und Spezialisierung aufgesplit- teten Ausbildungen vielfach auf statischen Lehrplänen beruhen, die nicht zukunftsfähige Health Professionals produzieren. Sie fordern deshalb eine neue Weltsicht und neue Formen des Lernens. Während vor 100 Jahren Flexner einem wissenschaftsbasierten Curriculum zum Durchbruch verhalf und in der Mitte des 20. Jahrhunderts das problembasierte Lernen ins Zentrum rückte, ist heute ein kompetenzbasiertes und systembezogenes Ausbildungssystem gefragt.

\section{Das Personal ist die wichtigste}

Ressource eines jeden

Gesundheitssystems.

Auf der institutionellen Ebene liegt der Schwerpunkt der Empfehlungen auf einer Förderung des systematischen gemeinsamen Lernens aller Berufsgruppen. Studierende sollen damit auf die interprofessionelle Teamarbeit und integrierte Prozesse vorbereitet werden. Die Ausbildungen sollen sich an den neuen Kompetenz-Standards ausrichten, auf lokaler Ebene alle Lernorte und Akteure einbeziehen und diese in globale Netzwerke der Zusammenarbeit einfügen. Auf der pädagogischen Ebene bedeutet dies, dass die heutigen institutionellen Grenzen der Ausbildung aufgegeben werden und kompetenzbasierte Curricula mit Lernfeldern entstehen, welche die lokalen Erfordernisse mit globalen Analysen zusammenbringen und die Informationstechnologie nutzen. Auf der Ebene der Steuerung sollen für die Akkreditierung die Standards neu formuliert werden.

Die Reformen beginnen mit einer neuen Denkweise, die die Herausforderungen wahrnimmt und zu lösen versucht. Wie bereits nach dem Erscheinen des Flexner-Reports vor 100 Jahren ist die Neuausrichtung ein langer und schwieriger Prozess, der neue Formen der Zusammenarbeit aller Beteiligten erfordert. 